PAPER

\title{
Qualitative neuropsychological performance characteristics in frontotemporal dementia and Alzheimer's disease
}

\author{
J C Thompson, C L Stopford, J S Snowden, D Neary
}

J Neurol Neurosurg Psychiatry 2005;76:920-927. doi: 10.1136/jnnp.2003.033779

See end of article for authors' affiliations

....................

Correspondence to: Jennifer Thompson Cerebral Function Unit, Greater Manchester Neuroscience Centre, Hope Hospital, Salford M6 8HD, UK; jennifer. thompson@manchester. ac.uk

Received 3 December 2003 In revised form 19 October 2004 Accepted 20 October 2004
Background: Frontotemporal dementia (FTD) and Alzheimer's disease are clinically distinct disorders, yet neuropsychological studies have had variable success in distinguishing them. A possible reason is that studies typically rely on overall accuracy scores, which may obscure differences in reasons for failure. Objectives: To explore the hypothesis that analysis of qualitative performance characteristics and error types, in addition to overall numerical scores, would enhance the neuropsychological distinction between FTD and Alzheimer's disease.

Methods: 38 patients with FTD and 73 with Alzheimer's disease underwent assessment of language, visuospatial abilities, memory, and executive function, using a neuropsychological screening instrument and standard neuropsychological tests. In each of these cognitive domains, performance characteristics and error types were documented, in addition to numerical scores on tests.

Results: Whereas comparison of neuropsychological test scores revealed some group differences, these did not occur consistently across tests within cognitive domains. However, analysis of performance characteristics and error types revealed qualitative differences between the two groups. In particular, FTD patients displayed features associated with frontal lobe dysfunction, such as concrete thought, perseveration, confabulation, and poor organisation, which disrupted performance across the range of neuropsychological tests.

Conclusions: Numerical scores on neuropsychological tests alone are of limited value in differentiating FTD and Alzheimer's disease, but performance characteristics and error types enhance the distinction between the two disorders. FTD is associated with a profound behavioural syndrome that affects performance on cognitive assessment, obscuring group differences. Qualitative information should be included in neuropsychological research and clinical assessments.
$\mathrm{F}$ rontotemporal dementia (FTD) is the most common clinical syndrome associated with frontotemporal lobar degeneration (FTLD). It is associated with a nonAlzheimer pathology that primarily affects the frontal and temporal cortex. Alterations in personality and behaviour are the most salient clinical features of FTD and are pivotal to the diagnostic criteria. ${ }^{12}$ This contrasts sharply with Alzheimer's disease, where the diagnosis is principally dependent on the presence of cognitive symptoms. ${ }^{3}$

Despite the striking behavioural abnormalities, cognitive impairment is also a central feature of FTD. Patients show "frontal" executive impairment and clinical criteria suggest that this is in the absence of severe amnesia, aphasia, or perceptuospatial disorder. ${ }^{124}$ This pattern differs from the cognitive changes observed in Alzheimer's disease-namely, deficits in episodic memory, language and perceptuospatial function. ${ }^{5}$ These cognitive differences are consistent with functional imaging studies that have shown anterior hemisphere abnormalities in FTD and posterior hemisphere abnormalities in Alzheimer's disease. ${ }^{6}$

Given the contrasting clinical profiles and patterns of CBF abnormality, one might expect direct neuropsychological comparison of FTD and Alzheimer's disease to yield a double dissociation, with FTD patients performing poorly on tests of executive function, but showing relatively preserved performance on tests of memory and perceptuospatial abilities, and Alzheimer patients showing the reverse pattern of findings. It is surprising, then, that comparative neuropsychological studies have produced inconsistent and sometimes counterintuitive findings. Some investigators have reported impaired performance on tests of perceptuospatial function in Alzheimer's disease relative to FTD, ${ }^{8-12}$ whereas others have reported no difference. ${ }^{13-17}$ Similarly, some studies have shown worse performance on tests of memory in Alzheimer's disease than in FTD, ${ }^{10}{ }^{14-16}{ }^{18}$ whereas others have found commensurate performance in both groups. ${ }^{8}{ }^{11-13}$ Greater impairment of executive function in FTD has been found by some investigators, ${ }^{11}{ }^{11-16}$ but a few studies have shown no difference between FTD and Alzheimer's disease on these tasks. ${ }^{12} 131719$

Differences in study design are likely to account for some of these conflicting results. However, the lack of clear differentiation between these two clinically distinct disorders may reflect the fundamental nature of neuropsychological assessment. Regardless of how well standardised and validated a neuropsychological instrument may be, there is no one to one correspondence between a specific test and a neuropsychological function. Tasks may certainly be sensitive to one aspect of cognition, but are never specific to that cognitive domain. For example, counting arrays of dots is a simple test of spatial function, included in a battery of tests designed to tap perceptual and spatial function. ${ }^{20}$ However, an inattentive, impulsive patient might also fail to count dots accurately for reasons of inattentiveness and lack of concern for accuracy. Thus a spatial task may be failed for executive reasons. Similarly, a putative test of executive function that makes spatial demands may be failed for spatial rather than executive reasons. It is possible, then, that FTD and

Abbreviations: FTLD, frontotemporal lobar degeneration; FTD, frontotemporal dementia; MMSE, mini mental state examination; SPECT, single photon emission tomography; VOSP, visual object and space perception 
Alzheimer patients may achieve similar scores on tasks for different underlying reasons.

Neuropsychological test scores are essentially summary statements about a fairly narrowly defined behavioural response. ${ }^{21}$ Despite being ostensibly objective, test scores rarely reveal information about how a particular task was carried out, or the types of error committed. Such information could shed light on the underlying reason for impairment on a given test. Consideration of qualitative aspects of test performance may be especially pertinent in FTD, given the behavioural abnormalities that characterise the condition, which include disinhibition, mental rigidity and inflexibility, concrete thought, distractibility, impersistence, economy of mental effort, and perseverative behaviour. ${ }^{22}$ It would be reasonable to suppose that such behavioural abnormalities would affect performance on tests across a variety of cognitive domains.

Our aim in this study was to examine both quantitative and qualitative aspects of neuropsychological test performance in a group of clinically diagnosed FTD and Alzheimer patients. We predicted that a greater memory and perceptuospatial disorder in Alzheimer's disease and a greater executive impairment in FTD would lead to some performance differences between the two groups on tests tapping these domains. However, we expected that such performance differences would not be consistent or clear cut, but that analysis of error types and performance characteristics would enhance the differentiation between FTD and Alzheimer's disease.

\section{METHODS}

\section{Patients}

The study group comprised 111 patients with a clinical diagnosis of Alzheimer's disease $(n=73)$ or frontotemporal dementia $(n=38)$. The Alzheimer's disease group contained a significantly greater proportion of female patients than the FTD group, which was more evenly matched in terms of sex (FTD, 16 female; 22 male; Alzheimer's disease, 45 female; 28 male $(p=0.047))$. Duration of symptoms did not differ significantly between the groups (mean (SD): FTD, 3.2 (2.2) years; Alzheimer's disease, 2.6 (1.6) years). The mini-mental state examination (MMSE) score was significantly higher in the FTD group (FTD, 22 (6); Alzheimer's disease, 18 (5) $(\mathrm{p}=0.002))$.

All patients underwent detailed history taking and neurological examination as part of their diagnostic work up. Patients were interviewed with their principal carer by a consultant neurologist in order to determine the evolution of their condition, with particular reference to symptoms arising from problems in language, visuospatial function, memory, and behaviour. The neurological examination was directed at eliciting signs of visuospatial disorientation, speech impairment, alterations in power, tone, and coordination, and the presence or absence of primitive reflexes. Diagnoses were made on the basis of historical information, clinical interview, and neurological examination, and were supported by findings on structural (magnetic resonance) and functional imaging (single photon emission computed tomography (SPECT)). All patients met currently accepted diagnostic criteria for FTD or Alzheimer's disease..$^{1-3}$ The cognitive and behavioural features are those that can be elicited from clinical interview.

\section{Inclusion criteria}

The study group comprised consecutive referrals to a regional diagnostic dementia clinic presenting between February 1999 and February 2003, in whom a full set of neuropsychological data was available. Only patients in whom a diagnosis of FTD or Alzheimer's disease was evident on the basis of clinical history and supported by neuroimaging were included in the study, to avoid the inherent circularity of defining patients on the basis of neuropsychology. The FTD group contained only individuals with the behavioural syndrome of frontotemporal dementia, and not semantic dementia or progressive nonfluent aphasia. Individuals with a mixed clinical syndrome were not included in the study.

\section{Evaluation}

Data collection was based on the patients' performance on neuropsychological tests administered as the time of their initial assessment. Each patient underwent evaluation using a locally constructed neuropsychological screening instrument, ${ }^{23}{ }^{24}$ tapping language, perception, spatial function, praxis, memory, and executive abilities. Examiners record both accuracy scores and qualitative aspects of performance, such as error types and behavioural characteristics of the patient. Neuropsychological profiles derived from the instrument are strongly related to the patterns of abnormality on SPECT $^{25}$ and, when combined with historical and neurological information, have yielded a $97 \%$ accuracy rate of clinical diagnosis in over 200 pathologically confirmed cases of dementia in this centre. In addition to the neuropsychological screening instrument, standardised neuropsychological tests of language and perceptuospatial function were also included in the initial assessment. The neuropsychological tests included in the present study were selected to represent language, perceptuospatial function, memory, and executive function. For each of these domains of cognitive function, performance characteristics and error types were recorded, in addition to numerical accuracy scores.

\section{Neuropsychological assessment}

As noted in the introduction, it is intrinsic to the nature of neuropsychological tests that they tap multiple cognitive functions. For example, presentation of line drawings can be used to assess visual perception, semantic access/knowledge, and naming. Similarly, presentation of photographs of celebrities can be used to assess visual perception, semantic access/knowledge, naming, and memory. For convenience, tests have been grouped under language, perceptuospatial function, memory, and executive function.

\section{Language}

Accuracy scores

- Graded naming test. Total naming score on the graded naming test $(/ 30)$.

- Boston naming test. Number of items named on a shortened version of this test $(/ 10)$.

- Naming famous faces. Ability to name famous faces taken from the entertainment media and political sphere (/9).

- Repetition. Ability to repeat monosyllabic and polysyllabic word strings and short phrases (/6).

- Sentence comprehension. Ability to interpret sentences correctly, based on individual word semantics, syntactic structure, and relational terms (/11).

- Metaphor interpretation. Ability to abstract meanings of common metaphors and proverbs (/4).

\section{Performance characteristics and error types}

- Graded naming test error types. Errors were categorised as follows: superordinate semantic category (for example, "animal" for kangaroo); coordinate semantic category ("koala" for kangaroo); phonological ("cuttleshock" for shuttlecock); functional description ("to open a bottle" for corkscrew); omission ("don't know"/no response); perceptual error ("glasses" for handcuffs); perseverative response (repetition of an earlier response). 
- Repetition error types. The following were recorded: omissions of words; word order errors; phonological errors; concrete responses (for example, an instruction to repeat "an uncommon animal" elicits the response "giraffe").

- Sentence comprehension. The presence or absence of concrete responses was recorded. (for example, the question "would you use a saw to cut wood?" elicits the response "I've got a gas fire so I don't need to cut wood.")

- Metaphor interpretation. The presence or absence of concrete explanations was recorded (for example, an interpretation of "every cloud has a silver lining" as "something to do with the weather").

\section{Perceptuospatial}

Accuracy scores

- Line drawing identification. Ability to provide appropriate identifying information for line drawings of objects taken from the Boston naming test $(/ 10)$.

- Celebrity identification. Ability to provide appropriate identifying information for famous faces taken from the entertainment media and political sphere (/9).

- Visual object and spatial perception battery. Total scores taken from the following subtests: screening; incomplete letters; silhouette perception; object decision; dot counting; position discrimination; number location; cube analysis.

- Visual construction. Ability to construct accurate copies of four line drawings: interlocking pentagons taken from the MMSE; a flower; a simple abstract design, and a simplified version of the Rey-Osterreth figure (/4).

\section{Performance characteristics and error types}

Qualitative assessment of perceptuospatial ability was limited by lack of consistency in reporting error types in some tests. Reliable data could be obtained from the visual construction test.

- Visual construction error types. The presence or absence of the following was recorded: spatial errors (that is, drawing individual elements of a figure in isolation or in incorrect spatial alignment), perseverative strokes (repeated redrawing of part of the figure in the same location; omission of detail; organisational/planning error (for example, individual elements drawn before overall outline leading to poor "fit" or leaving insufficient space on the page to complete design; overelaboration of detail (such as addition of extra details not present in the original). Attribution of error type was based on evaluation of drawings in addition to written report of the original neuropsychological assessor.

\section{Memory}

Accuracy scores

- Immediate memory. Forward and reverse digit span.

- Story recall. Number of elements recalled from a short fable-like story. Free and cued recall was assessed immediately after presentation and again following a 30 minute delay. The proportion of information retained following a delay was calculated by dividing delayed recall by immediate recall.

- Celebrity recall. Photographs of celebrities were presented as part of the language/perception assessment. Free recall of these celebrities was assessed 30 minutes after presentation, by means of naming or provision of identifying information (/9).

\section{Performance characteristics and error types}

- Story recall error types. The presence or absence of the following was recorded: intrusions (responses irrelevant to the task relating to an earlier test); confabulation (introduction of fictional elaborative elements in recall); misconstruction (misordering or misconstruing elements resulting in a confused, disordered account).

\section{Executive functions \\ Accuracy scores}

- Category fluency. Number of animal names produced in one minute.

- Letter fluency. Number of words beginning with " $F$ " produced in one minute. Proper nouns and derivations of the same word stem (for example, fill, filling, filler) were not admissible.

- Weigl's test. Grouping a set of blocks according to colour, shape, and motif. Three points were awarded for each correct uncued sort, two points if the patient could complete the sort following a partial grouping by the examiner, and one point if the patient succeeded in identifying the sorting dimension following a complete sort by the examiner (/9).

\section{Performance characteristics and error scores}

- Verbal fluency error types. The number of repetitions and rule violations was recorded (for example, production of a proper noun in the letter fluency task).

- Weigl's test errors. The presence or absence of: perseveration of a particular sorting category; dismantling of the blocks when partially/completely sorted by the examiner and regrouping according to a previously relevant dimension, or idiosyncratic sorting rules.

\section{Behavioural observations}

Examiners' reports comprise a written description of patients' behaviour during the assessment, in which it is noted whether their behaviour was socially appropriate, whether they cooperated with the assessment, and whether they engaged in tests with appropriate effort and concern for accuracy and maintained attention. Conversely, it is also noted if patients are easily distractible, are disinhibited, or show economy of effort-that is, if they give rapid, cursory responses to questions or abandon tests readily. For the purposes of this study, the presence or absence of social appropriateness, disinhibited behaviour, and economy of effort was recorded.

\section{Procedure}

Two independent raters, JT and CS, documented numerical accuracy scores, performance characteristics, and error types based on raw test data and documentation in clinical reports.

\section{Statistical analysis}

Data analyses were carried out using SPSS (version 10) and StatsDirect (version 2.3.7). Logistic regression analyses with clinical diagnosis as the dichotomous outcome variable $(\mathrm{FTD}=1 /$ Alzheimer's disease $=0 ;$ classification cut off point $=0.5$ ) was used to calculate the degree to which individual items yielded an increase or a decrease in the odds of the diagnosis being FTD as opposed to Alzheimer's disease. Increases or decreases in odds were considered statistically significant at $\mathrm{p}<0.05$. Continuity corrections were applied to the data where items were only present in one or other group. Items that yielded a significant increase or decrease in odds were entered into further logistic regression analyses to determine the predictive value of quantitative and qualitative 
data. The frequency distributions of the two groups of patients according to the examiners' behavioural observations were compared using $\chi^{2}$ tests.

\section{RESULTS}

\section{Neuropsychological assessment Language}

Total scores on the graded naming test were of no value in predicting diagnosis (table 1). However, error responses were of significant value: phonological errors were predictive of Alzheimer's disease, whereas perseverative responses increased the probability of a FTD diagnosis.

Naming of either line drawings from the Boston naming test or celebrities did not differentiate the groups. Overall accuracy on a test of repetition was commensurate, but the presence of concrete errors significantly increased the likelihood of an FTD diagnosis, while phonological errors increased the likelihood of an Alzheimer diagnosis. FTD patients' performance was poorer on sentence comprehension and metaphor interpretation. The presence of concrete errors on these tasks was a significant predictor of FTD.

\section{Perceptuospatial}

The ability to identify line drawings or celebrities did not differentiate the groups (table 2). On the visual object and space perception (VOSP) battery, Alzheimer patients showed poorer performance on some subtests (incomplete letters, position discrimination, cube analysis), but not others (silhouette identification, object decision, dot counting, number location). On a visual construction task, Alzheimer patients produced fewer correct drawings. There were also differences in the type of errors committed. A large proportion of Alzheimer patients made spatial errors, whereas none of the FTD patients did. Thus spatial errors were highly predictive of Alzheimer's disease. By contrast, organisational errors, perseverations, or overelaborated copies were significant predictors of FTD. Figure 1 shows examples of drawings completed by the two patient groups. The copies carried out by an FTD patient showed poor organisation but preserved spatial configuration. By contrast, the copies carried out by an Alzheimer patient showed poor spatial configuration, with individual elements drawn in isolation.

\section{Memory}

Alzheimer patients were impaired in story recall (both immediate and delayed) and celebrity recall relative to FTD patients (table 3 ). Of note was that there was a marked difference in the amount of information retained over a delay in story recall. In the Alzheimer group, patients recalled a median of $0 \%$ of the information produced on immediate recall, whereas FTD patients produced $100 \%$ of their original recall. There were also qualitative differences between the groups: misconstructed or confabulatory accounts significantly increased the odds that the patient had FTD, whereas intrusions of unrelated information increased the odds that the patient had Alzheimer's disease.

\section{Executive functions}

On a letter fluency task, FTD patients produced fewer correct responses and made more rule violations, although this was not statistically significant (table 4). Alzheimer patients produced more repetition errors on a category fluency test. Overall scores on the Weigl's test did not differentiate the groups. However, when individuals dismantled blocks partially sorted by the examiner, and regrouped them perseveratively this increased the likelihood of an FTD diagnosis.

\section{Effect of dementia severity}

In order to ensure that the observed pattern of findings did not reflect the inclusion of more severely affected individuals,

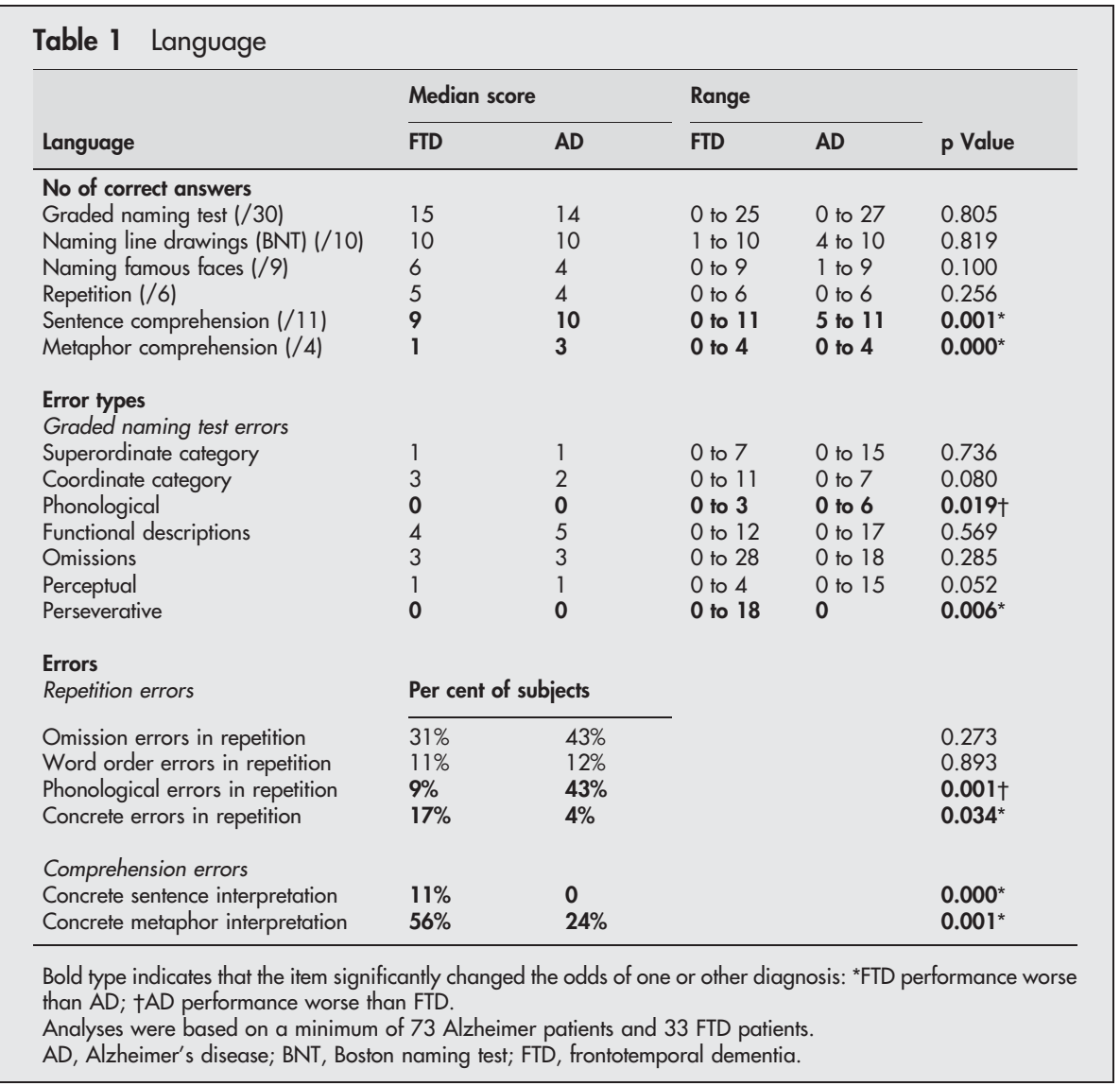


FTD
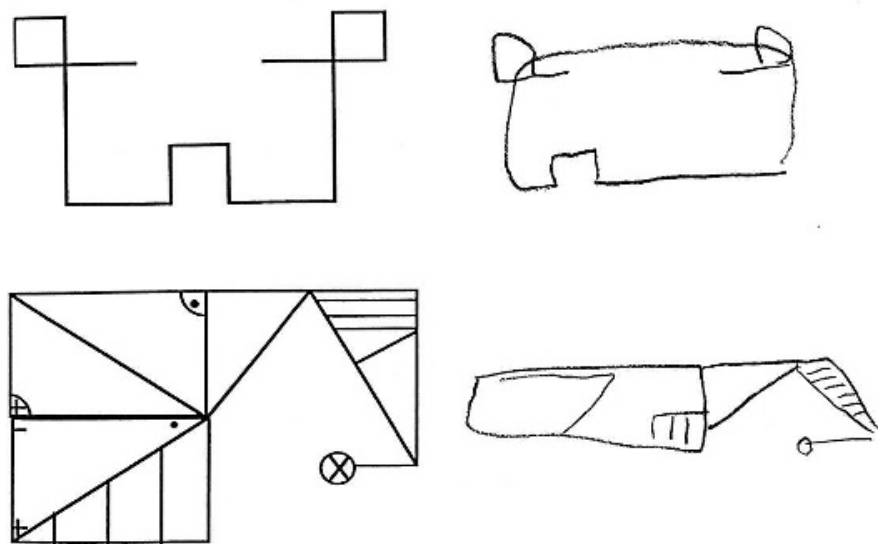

$A D$

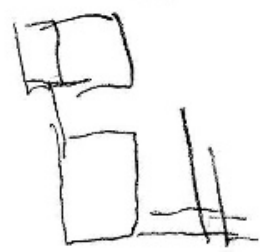

Figure 1 Examples of visual construction in frontotemporal dementia (FTD) and Alzheimer's disease (AD). the analyses were repeated excluding those with MMSE scores of 15 and under, leaving 48 Alzheimer and 29 FTD patients. Although the pattern of results was broadly similar, a few differences emerged. The number of line drawings from the Boston naming test now differentiated the groups $(p=0.045)$, with Alzheimer patients naming fewer than FTD patients. Overall score on the Weigl's test also differentiated the groups $(\mathrm{p}=0.033)$, with FTD patients achieving lower scores than Alzheimer patients.

The majority of tests and qualitative features remained statistically significant; however, the following no longer differentiated the groups: number of phonological errors on the graded naming test, and the presence of perseverative strokes and poor organisation on visual construction.

Predictive value of quantitative and qualitative data A logistic regression analysis was conducted to determine the statistical value of numerical scores and error types in predicting diagnosis. Items that significantly increased or decreased the odds of FTD as opposed to Alzheimer's disease were included in this analysis. Items that only occurred in one or other of the patient groups were excluded from the analysis to avoid statistical overfitting (in visual construction, spatial errors occurred only in the Alzheimer's disease group and overelaboration occurred only in the FTD group; in repetition, concrete errors occurred only in the FTD group). Items from the VOSP were excluded from this analysis as, because of time constraints at the time of test administration, data were not available for a relatively large number of patients. Although immediate and delayed free and cued recall of a story all reached significance, only immediate and delayed free recall were retained in order to reduce the number of variables in the analysis.

A logistic regression analysis with diagnosis as the dichotomous outcome variable $(\mathrm{FTD}=1$ /Alzheimer's disease $=0$ ), and a classification cut off point of 0.5 was carried out in two stages, the first involving quantitative test scores only and the second with the inclusion of qualitative

Table 2 Perceptuospatial function

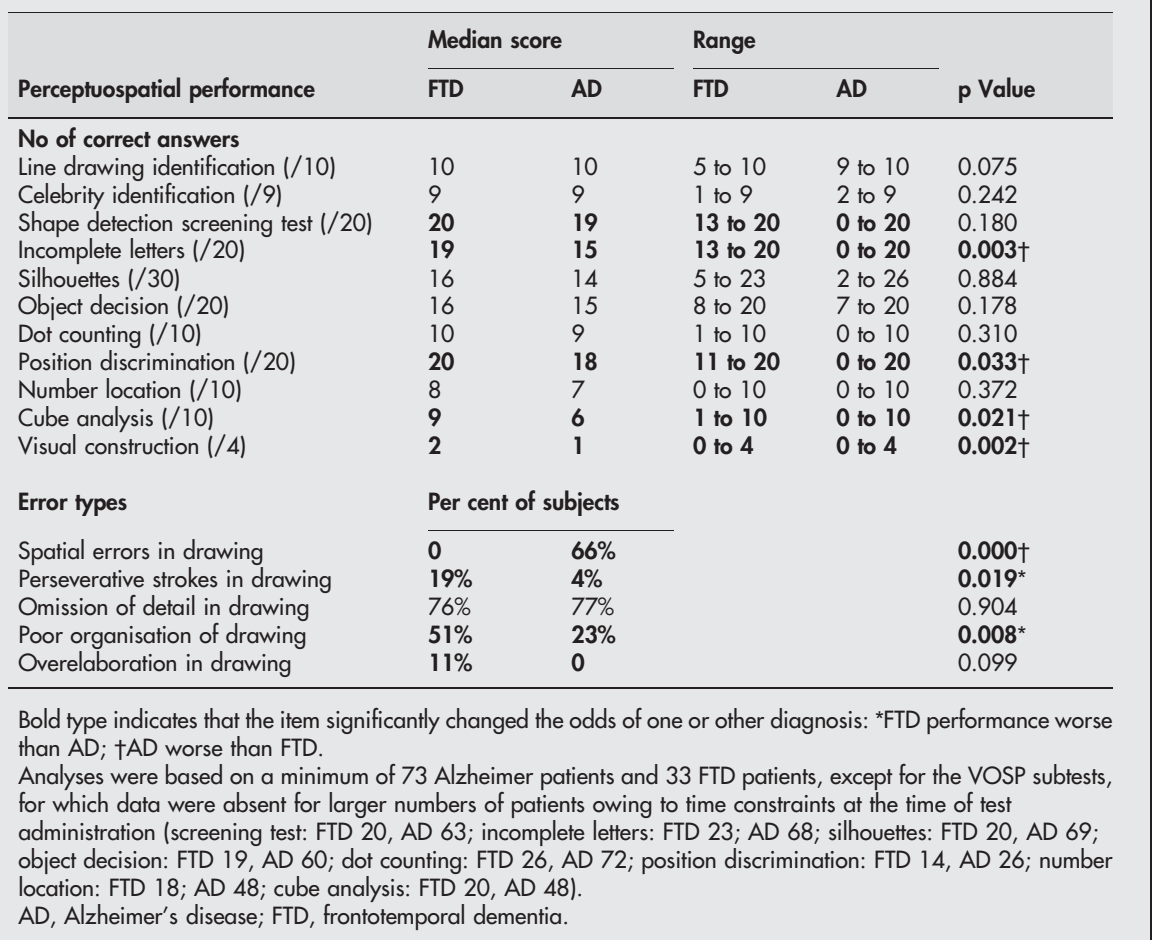




\begin{tabular}{|c|c|c|c|c|c|}
\hline \multirow[b]{2}{*}{ Memory } & \multicolumn{2}{|c|}{ Median score } & \multicolumn{2}{|l|}{ Range } & \multirow[b]{2}{*}{$p$ Value } \\
\hline & FTD & AD & FTD & AD & \\
\hline \multicolumn{6}{|l|}{ No of correct answers } \\
\hline Digit span forwards & 6 & 6 & 0 to 6 & 2 to 9 & 0.954 \\
\hline Digit span backwards & 3 & 3 & 0 to 10 & 0 to 7 & 0.601 \\
\hline Story: free recall & 5 & 2 & 0 to 10 & 0 to 10 & $0.007 \dagger$ \\
\hline $\begin{array}{l}\text { Story: cued recall } \\
\text { Story: delayed free recall }\end{array}$ & 7 & 3 & 0 to 10 & 0 to 10 & $\begin{array}{l}0.001 \dagger \\
0.000+\end{array}$ \\
\hline Story: delayed cued recall & & & & & $0.000+$ \\
\hline Story: \% retained with delay (free) & 100 & 0 & 0 to $175 \% \ddagger$ & 0 to $400 \% \ddagger$ & $0.000+$ \\
\hline Story: \% retained with delay (cued) & 97 & 50 & 0 to $160 \% \ddagger$ & 0 to $400 \% \ddagger$ & $0.001 \dagger$ \\
\hline Celebrity recall (/9) & 4 & 1 & 0 to 8 & 0 to 9 & $0.000+$ \\
\hline Error types & \multicolumn{2}{|c|}{ Per cent of subjects } & & & \\
\hline Intrusions in story recall & 6 & 31 & & & $0.012 \dagger$ \\
\hline Confabulations in story recall & 24 & 8 & & & $0.038^{*}$ \\
\hline Misconstruction in story recall & 50 & 9 & & & $0.000^{*}$ \\
\hline \multicolumn{6}{|c|}{$\begin{array}{l}\text { Bold type indicates that the item significantly changed the odds of one or other diagnosis: *FTD performance worse } \\
\text { than } A D ;+A D \text { performance worse than FTD. } \\
\text { Analyses were based on a minimum of } 73 \text { Alzheimer's disease patients and } 33 \text { FTD patients. } \\
\text { †Delayed recall is expressed as a percentage of immediate recall; figures are greater than } 100 \% \text { where the amount } \\
\text { of information produced on delayed recall exceeds that produced on immediate recall. } \\
A D \text {, Alzheimer's disease; FTD, frontotemporal dementia. }\end{array}$} \\
\hline
\end{tabular}

characteristics. The following quantitative variables were entered into the first stage of analysis: sentence repetition, metaphor interpretation, visual construction, recall of celebrities, and free recall of a story (immediate and delayed). The resulting logistic regression model correctly classified $71 \%$ of FTD patients and $93 \%$ of Alzheimer patients, yielding a sensitivity of 0.71 and a specificity of 0.96 .

A composite score for this regression algorithm was created and entered into the next stage of the logistic regression analysis along with the following qualitative variables: phonological errors on the graded naming test; errors of phonology and concreteness in sentence repetition; concrete errors in metaphor interpretation; errors of perseveration and organisation in copying drawings; intrusions, confabulations and misconstructions in story recall; and dismantling on the Weigl's test. The purpose of entering a composite score of the "quantitative" logistic regression equation instead of the individual items was to minimise the number of variables in the analysis. The resulting logistic regression model correctly classified $96 \%$ of FTD patients and 93\% of Alzheimer patients, yielding a sensitivity of 0.91 and a specificity of 0.96 .

\section{Behavioural observations}

Ninety one per cent of Alzheimer patients were noted to behave appropriately during the assessment-that is, they were cooperative and engaged in tests with normal concern for accuracy-compared with only $30 \%$ of FTD patients $(\mathrm{p}<0.0005) ; 56 \%$ of FTD patients showed disinhibited behaviour compared with just $4 \%$ of Alzheimer patients $(\mathrm{p}<0.0005)$; and $35 \%$ of FTD patients were recorded as showing economy of effort when carrying out tasks compared with $3 \%$ of Alzheimer patients $(\mathrm{p}<0.0005)$.

\section{DISCUSSION}

We predicted that neuropsychological test scores alone would only be partially successful in distinguishing FTD and Alzheimer's disease because test scores mask differences in underlying reason for test failure. In contrast, we predicted that analysis of qualitative aspects of performance would enhance the differentiation between the two groups and highlight clinically distinct patterns of performance.

Some overall test scores, as predicted, did differentiate the groups: FTD patients performed worse on a letter fluency test

Table 4 Executive function

\begin{tabular}{|c|c|c|c|c|c|}
\hline \multirow[b]{2}{*}{ Executive function } & \multicolumn{2}{|c|}{ Median score } & \multicolumn{2}{|l|}{ Range } & \multirow[b]{2}{*}{ p Value } \\
\hline & FTD & $A D$ & FTD & $A D$ & \\
\hline $\begin{array}{l}\text { No of correct answers } \\
\text { Category fluency } \\
\text { Letter fluency } \\
\text { Weigl's test } / 9\end{array}$ & $\begin{array}{l}10 \\
4 \\
4\end{array}$ & $\begin{array}{l}9 \\
7 \\
6\end{array}$ & $\begin{array}{l}0 \text { to } 26 \\
0 \text { to } 18 \\
0 \text { to } 9\end{array}$ & $\begin{array}{l}2 \text { to } 21 \\
1 \text { to } 29 \\
0 \text { to } 9\end{array}$ & $\begin{array}{l}0.762 \\
0.016^{\star} \\
0.157\end{array}$ \\
\hline $\begin{array}{l}\text { Error types } \\
\text { Letter fluency: repetitions } \\
\text { Letter fluency: rule violations } \\
\text { Category fluency: repetitions } \\
\text { Category fluency: rule violations }\end{array}$ & $\begin{array}{l}0 \\
0 \\
0 \\
0\end{array}$ & $\begin{array}{l}0 \\
0 \\
0 \\
0\end{array}$ & $\begin{array}{l}0 \text { to } 3 \\
0 \text { to } 3 \\
0 \text { to } 4 \\
0 \text { to } 1\end{array}$ & $\begin{array}{l}0 \text { to } 4 \\
0 \text { to } 3 \\
0 \text { to } 9 \\
0 \text { to } 4\end{array}$ & $\begin{array}{l}0.451 \\
0.096 \\
0.036 \dagger \\
0.785\end{array}$ \\
\hline & \multicolumn{2}{|c|}{ Per cent of subjects } & & & \\
\hline $\begin{array}{l}\text { Perseveration on Weigl's test } \\
\text { Dismantling on Weigl's test }\end{array}$ & $\begin{array}{l}44 \\
33\end{array}$ & $\begin{array}{l}29 \\
15\end{array}$ & & & $\begin{array}{l}0.117 \\
0.031^{*}\end{array}$ \\
\hline
\end{tabular}

Bold type indicates that the item significantly changed the odds of one or other diagnosis. *FTD performance worse than $A D ; \dagger A D$ performance worse than FTD.

Analyses were based on a minimum of 73 Alzheimer's disease patients and 33 FTD patients.

$A D$, Alzheimer's disease; FTD, frontotemporal dementia. 
and on sentence and metaphor comprehension than Alzheimer patients. By contrast, Alzheimer patients performed worse on drawing tests, on some subtests of a perceptuospatial battery, and on tests of memory. These findings are entirely in keeping with clinical observations that FTD predominantly affects frontal executive skills, whereas in Alzheimer's disease there is prominent involvement of memory and perceptuospatial abilities. They are in keeping too with neuroimaging findings, which indicate that FTD is a disorder of the anterior hemispheres, whereas Alzheimer's disease involves the medial temporal lobes and posterior hemispheres. ${ }^{67}$ Nevertheless, as expected, despite the evident clinical differences between the two groups, performance on executive and perceptuospatial tasks did not consistently differentiate the groups. Category fluency and performance on the Weigl's test did not differentiate the groups (although the latter did reach significance when patients with MMSE scores of 15 or under were excluded from the analysis). Similarly, comparable performance was elicited on some of the perceptual and spatial tests, including the simple dot counting task. Comparable performance occurred despite the fact that the Alzheimer patients showed no behavioural features to suggest frontal lobe disorder, and the FTD patients had no history of difficulty negotiating or becoming lost in the environment to suggest spatial disorder.

Our analysis of performance characteristics and error types showed that even when test scores did not differentiate the two groups, there are qualitative differences in performance, supporting the notion that failure arises for different underlying reasons. The groups were not differentiated by their total scores on a standard naming test, yet certain error types were strongly predictive of one or other diagnosis: perseverative errors increased the probability of FTD, whereas phonological errors were predictive of Alzheimer's disease. Overall repetition scores did not differentiated the two groups but error types did: the presence of concrete responses increased the odds of a FTD diagnosis, and phonological errors were predictive of an Alzheimer's disease diagnosis. Overall scores on the Weigl's test did not differentiate the groups, yet the FTD patients were more likely to show "dismantling" behaviour. That is, they failed to identify the sorting rule when blocks were grouped by the examiner, but dismantled the blocks, rearranging them according to a previous criterion or their own idiosyncratic sorting method. Such behaviour indicates an inability to shift mental set. In category fluency, repetition of early items-suggesting an inability to remember items previously produced-was a significant predictor of Alzheimer's disease.

A logistic regression analysis indicated that both numerical scores and error types were of value in predicting the diagnosis of individual cases. When test scores only were entered into the analysis, the resultant logistic regression model correctly classified $87 \%$ of patients. However, the accuracy of this classification was not equal between the two groups. Test scores correctly classified 93\% of Alzheimer patients, but just $71 \%$ of FTD patients. When error types were added to the analysis, the classification accuracy remained unchanged for Alzheimer's disease, but increased to $96 \%$ for FTD. This suggests that although test scores are of considerable statistical value in predicting diagnosis, the inclusion of error types in addition to test scores greatly enhances the differentiation of the two conditions.

Across the range of tasks, features that are typically associated with frontal lobe failure were predictive of FTD: concreteness of thought, perseveration, confabulation and misconstruction, and poor organisation. Concrete responses occurred across various tasks, including interpretation of metaphor and proverb, a task often used to measure the capacity for abstract thought. Concrete responses also occurred on tasks that ostensibly may not be thought to require abstract thought: interpretation of complex sentences and a simple task of repetition. These findings indicate that concrete and overliteral responses are a salient characteristic of FTD. Perseveration on naming and drawing tests significantly increased the odds of an FTD diagnosis. On the Weigl's test of abstraction and set shifting, the "dismantling" form of perseveration previously described increased the odds of an FTD diagnosis. Confabulations and misconstructions were features of FTD patients' memory performance. Of note was also that the amount of information retained over a delay varied considerably between the two groups: FTD patients retained a median of $100 \%$ of their immediate recall compared to $0 \%$ in the Alzheimer group. These qualitative features strongly suggest a "frontal" form of memory loss in FTD, contrasting with an amnesia secondary to medial temporal lobe pathology in Alzheimer's disease. In FTD, drawing errors most commonly arose because of organisational failure. Frankly spatial errors were notably absent in FTD.

The relatively high rate of phonological errors observed in the Alzheimer group deserves comment, as it is a generally held view that phonological aspects of language are well preserved in Alzheimer's disease. Phonological errors on the graded naming test significantly increased the probability of an Alzheimer's disease diagnosis. However, when analysis was restricted to those patients scoring 15 or more on the MMSE this did not reach significance, suggesting that this finding reflected the inclusion of individuals with more severe cognitive impairment. Nevertheless, on a test of word and sentence repetition, $43 \%$ of Alzheimer patients made phonological errors, compared with just $9 \%$ of FTD patients. This feature remained highly predictive of Alzheimer's disease when analysis was restricted to those scoring over 15 on the MMSE. Thus repetition appears to be a particularly sensitive task in eliciting phonological impairment. Our findings are consistent with other published reports. Alzheimer patients have been reported to make no more phonological errors than healthy controls in spontaneous speech. ${ }^{26}$ By contrast, on repetition tasks Alzheimer patients are more prone to committing phonological errors, ${ }^{27}$ the rate of which appears to be related to the phonological and syntactic complexity of the material used..$^{28}$

Our data enable us to resolve discrepancies in published reports. Previous neuropsychological studies have not always shown significant differences between FTD and Alzheimer's disease. Furthermore, there has been inconsistency regarding the neuropsychological measures reported to differentiate the two conditions. We have shown that neuropsychological test scores can actually obscure differences between patient groups because they mask the underlying reasons for test failure. In FTD there is profound behavioural disturbance, characterised by impulsive and disinhibited behaviour, perseveration, impersistence, inattention, and economy of effort. These behaviours affect test performance in a way that is not apparent, and is actually disguised when numerical scores alone are considered.

FTD is characterised behaviourally by a "frontal lobe" syndrome. By contrast, patients with Alzheimer's disease are usually socially appropriate and do not generally display behaviours suggestive of frontal lobe dysfunction. In the present study, the performance of Alzheimer patients was not coloured by perseveration, concrete thought, confabulation, or economy of effort, and their behaviour was generally appropriate during testing. Nevertheless, there were few quantitative differences between FTD and Alzheimer patients on executive tasks. The finding that Alzheimer patients perform similarly to FTD patients in terms of their overall scores, despite lacking a "frontal lobe" syndrome, suggests that they are failing these tasks for non-frontal reasons. Executive tasks are complex and make demands on multiple 
cognitive abilities including language, working memory, and often spatial abilities. Weigl's test requires the ability to perceive an array of blocks and evaluate any differences between them. To carry out this task efficiently requires intact visual perception and spatial abilities, functions that are compromised in Alzheimer's disease. Similarly, the impairment of short term memory characteristic of Alzheimer's disease may result in a patient sorting blocks according to a previous sorting rule because of failure to keep track, and not because of a "frontal" form of executive dysfunction.

Some methodological issues merit discussion. The patients involved in the study were consecutive referrals to a specialist dementia clinic, providing a representative sample of each patient group, covering a range of illness severities. Consequently, patients were not matched for age, sex, or severity of illness. It has been customary when conducting research with individuals who have degenerative neurological disorders to match patient groups for dementia severity according to their scores on the MMSE. However, we did not believe this was appropriate because this bedside test is heavily weighted towards memory impairment, with 16 of the 30 points allocated to memory items. Given the prominent memory disorder associated with Alzheimer's disease, matching across the two patient groups for MMSE scores in an attempt to yield groups of equivalent overall severity could not be meaningfully achieved. Indeed, predictably, scores on the MMSE were significantly lower in Alzheimer's disease than in FTD. Moreover, a notional "severity of dementia" is most unlikely to account for our findings, as the FTD group was more impaired on some measures, whereas the Alzheimer group was more impaired on others. The duration of symptoms was broadly equivalent in each group, so in this regard the groups were well matched.

Our study strongly indicates that reliance on numerical scores alone does not reliably differentiate FTD and Alzheimer's disease. FTD is associated with a profound behavioural disorder that affects performance on all cognitive tests, obscuring qualitative group differences. When performance characteristics and error types are properly taken into account, the accuracy of differentiation is enhanced. Knowledge of the performance characteristics and error types that characterise FTD ought to be of significant value to clinicians and may assist in the differential diagnosis of this condition and in the evaluation of individual patients. The study attests to the value of obtaining qualitative information in general neuropsychological research. Test scores must be viewed and interpreted in the context of both cognitive and behavioural performance to avoid misconceptions that can arise from a purely quantitative assessment. The latter generates "scores", which can serve to obscure rather than highlight significant qualitative differences between patients with different forms of dementia. These characteristic distinctions are clearly manifest in the clinical history of cognitive and behavioural symptoms and the findings of the neurological examination, corresponding to the two distinct clinical neuropsychological profiles of FTD and Alzheimer's disease.

\section{ACKNOWLEDGEMENTS}

We thank Andy Vail, lecturer in the Biostatistics Group, University of Manchester, for statistical advice.

\section{Authors' affiliations}

J C Thompson, C L Stopford, J S Snowden, D Neary, Cerebral Function Unit, Greater Manchester Neuroscience Centre, Hope Hospital, Salford, UK

Competing interests: none declared

\section{REFERENCES}

1 The Lund and Manchester Groups. Consensus Statement. Clinical and neuropathological criteria for fronto-temporal dementia. I Neurol Neurosurg Psychiatry 1994;4:416-18.

2 Neary D, Snowden JS, Gustafson L, et al. Frontotemporal lobar degeneration. A consensus on clinical diagnostic criteria. Neurology 1998;51:1546-54.

3 McKhann G, Drachman D, Folstein M, et al. Clinical diagnosis of Alzheimer's disease: report of the NINCDS-ADRDA work group under the auspices of Department of Health and Human Services Task Force on Alzheimer's disease. Neurology 1984;34:939-44.

4 Neary D, Snowden JS, Northern B, et al. Dementia of frontal lobe type. J Neurol Neurosurg Psychiatry 1988;1 1:592-8.

5 Cummings JL, Benson DF. Dementia. A clinical approach. Boston: Butterworth Heinemann, 1992.

6 Talbot PR, Snowden JS, Lloyd JJ, et al. The contribution of single photon emission tomography to the clinical differentiation of degenerative cortical brain disease. J Neurol 1995;242:579-86.

7 Talbot PR, Lloyd JJ, Snowden JS, et al. A clinical role for ${ }^{99 m T c-H M P A O ~ S P E C T ~}$ in the investigation of dementia? J Neurol Neurosurg Psychiatry 1998;64:306-13.

8 Elfgren C, Brun A, Gustafson L, et al. Neuropsychological tests as discriminators between dementia of Alzheimer type and frontotemporal dementia. Int J Geriatr Psychiatry 1994;9:635-42.

9 Mendez MF, Cherrier M, Perryman KM, et al. Frontotemporal dementia versus Alzheimer's disease: differential cognitive features. Neurology 1996;47:1 189-94.

10 Hodges JR, Patterson K, Ward R, et al. The differentiation of semantic dementia and frontal lobe dementia (temporal and frontal variants of frontotemporal dementia) from early Alzheimer's disease: a comparative neuropsychological study. Neuropsychology 1999;13:31-40.

11 Razani J, Brauer-Boone K, Miller B, et al. Neuropsychological performance of right and left frontotemporal dementia compared to Alzheimer's disease. J Int Neuropsychol Soc 2001;7:468-80.

12 Rascovsky K, Salmon DP, Ho GJ, et al. Cognitive profiles differ in autopsy confirmed frontotemporal dementia and Alzheimer's disease. Neurology 2002;58:1801-8.

13 Pachana NA, Brauer-Boone K, Miller B, et al. Comparison of neuropsychological functioning in Alzheimer's disease and frontotemporal dementia. J Int Neuropsychol Soc 1996;2:505-10.

14 Lindau M, Almkvist O, Johansson SE, et al. Cognitive and behavioural differentiation of frontal lobe degeneration of the non-Alzheimer type and Alzheimer's disease. Dement Geriatr Cogn Disord 1998;9:205-13.

15 Perry RJ, Hodges JR. Differentiating frontal and temporal variant frontotemporal dementia from Alzheimer's disease. Neurology 2000;54:2277-84

16 Mathuranath PS, Nestor PJ, Berrios GE, et al. A brief cognitive test battery to differentiate Alzheimer's disease and frontotemporal dementia. Neurology 2000;55:1613-20.

17 Grossi, D, Fragassi NA, Chiacchio L, et al. Do visuospatial and constructional disturbances differentiate frontal variant of frontotemporal dementia and Alzheimer's disease? An experimental study of a clinical belief. Int J Geriatr Psychiatry 2002;17:641-8.

18 Gregory CA, Orrell M, Sahakian B, et al. Can frontotemporal dementia and Alzheimer's disease be differentiated using a brief battery of tests? Int J Geriatr Psychiatry 1997; 12:375-83.

19 Kumar A, Schapiro MB, Haxby JV, et al. Cerebral metabolic and cognitive studies in dementia with frontal lobe behavioural features. J Psychiatr Res 1990;24:97-109.

20 Warrington EK, James M. Visual object and space perception battery, Bury St. Edmunds: Thames Valley Test Company, 1991

21 Lezak M. Neuropsychological assessment. Oxford: Oxford University Press, 1995.

22 Bathgate D, Snowden JS, Varma A, et al. Behaviour in frontotemporal dementia, Alzheimer's disease and vascular dementia. Acta Neurol Scand 2001;103:367-78.

23 Neary D, Snowden JS, Bowen DM, et al. Neuropsychological syndromes in presenile dementia due to cerebral atrophy. J Neurol Neurosurg Psychiatry 1986;49: 1630-74.

24 Snowden JS, Mann DM, Neary D. Distinct neuropsychological characteristics in Creutzfeldt-Jakob disease. J Neurol Neurosurg Psychiatry 2002;73:686-94

25 Neary D, Snowden JS, Sheilds RA, et al. Single photon emission tomography using $99 \mathrm{mTc}-\mathrm{HM}-\mathrm{PAO}$ in the investigation of dementia. I Neurol Neurosurg Psychiatry 1987;50:1101-9.

26 Blanken G, Dittman J, Haas JC, et al. Spontaneous speech in senile dementia and aphasia: implications for a neurolinguistic model of language production. Cognition 1987; 27:247-74.

27 Glosser G, Kohn, SE, Friedman RB, et al. Repetition of single words and nonwords in Alzheimer's disease. Cortex 1997;33:653-66.

28 Biassou N, Grossman M, Onishi K, et al. Phonologic processing deficits in Alzheimer's disease. Neurology 1995;45:2165-9.

29 Glosser G, Friedman RB, Kohn SE, et al. Cognitive mechanisms for producing nonwords: evidence from Alzheimer's disease. Brain Language 1998;63:32-49. 